\title{
Erratum to: Inhibition of human gamma delta T-cell antitumoral activity through HLA-G: implications for immunotherapy of cancer
}

Emilie Lesport · Jeremy Baudhuin - Sylvie Sousa - Joel LeMaoult • Alessia Zamborlini $\cdot$ Nathalie Rouas-Freiss $\cdot$ Edgardo D. Carosella Benoit Favier

Published online: 8 June 2011

(C) Springer Basel AG 2011

\section{Erratum to: Cell Mol Life Sci}

DOI 10.1007/s00018-011-0632-7

In the original version of the article, an error occurred in the article title. The correct title should read as:

Inhibition of human gamma delta T-cell antitumoral activity through HLA-G: implications for immunotherapy of cancer

The online version of the original article can be found under doi:10.1007/s00018-011-0632-7.

E. Lesport $\cdot$ J. Baudhuin $\cdot$ S. Sousa $\cdot$ J. LeMaoult .

N. Rouas-Freiss · E. D. Carosella · B. Favier $(\bowtie)$

CEAEA, I2BM, Service de Recherches en Hemato-Immunologie, 75475 Paris, France

e-mail: benoit.favier@cea.fr

E. Lesport $\cdot$ J. Baudhuin $\cdot$ S. Sousa $\cdot$ J. LeMaoult .

N. Rouas-Freiss · E. D. Carosella · B. Favier

UMR_E, Université Paris Diderot,

Institut Universitaire d'Hématologie, Paris, France

\section{A. Zamborlini}

CNRS UMR7212, Inserm U944, Université Paris Diderot,

Institut Universitaire d'Hématologie, Paris, France

E. Lesport · J. Baudhuin · S. Sousa - J. LeMaoult ·

A. Zamborlini - N. Rouas-Freiss - E. D. Carosella $\cdot$ B. Favier CEA, I2BM, Service de Recherches en Hemato-Immunologie, Institut Universitaire d'Hematologie, Hopital Saint Louis,

1 Avenue Claude Vellefaux, 75010 Paris, France 